\title{
Sensitive detection of Nitrogen Dioxide using gold nanoparticles decorated Single Walled Carbon Nanotubes
}

\author{
Sunil Kumar ${ }^{1}$, Vladimir Pavelyev ${ }^{1}$, Prabhash Mishra ${ }^{1}$, Nishant Tripathi ${ }^{1}$ \\ ${ }^{1}$ Samara National Research University, 34 Moskovskoe Shosse, 443086, Samara, Russia
}

\begin{abstract}
The modification of carbon nanotubes (CNTs) could enhance their surface and electric properties. To this purpose, we explore the impact of a thin layer of gold $(\mathrm{Au})$ on the surface of single wall carbon nanotubes (SWCNTs). SWCNTs have been grown by Chemical Vapor Deposition (CVD) method and decorated with gold nanoparticles were investigated as gas sensitive materials for detecting nitrogen dioxide $\left(\mathrm{NO}_{2}\right)$ at room temperature. Surface morphology and microstructure of Au-SWCNT have been characterized by FE-SEM and Raman Spectroscopy. Using the present collective approaches, the improvement in the detection of $\mathrm{NO}_{2}$ gas using Au-modified nanotubes is explained. However, Au-modified SWCNT gas sensors exhibited better performances compared to pristine SWCNTs. These changes in resistance and the shift of the Fermi level just after $\mathrm{NO}_{2}$ exposure was probably due to adsorption of $\mathrm{NO}_{2}$ molecules on the surface of Au-SWCNTs. Surface modification of nanotubes with understanding of sensing ability at atomic level opens the new way to design a selectivity gas sensor.
\end{abstract}

Keywords: carbon nanotubes; nanostructured materials; nanotechnology; functionalization; sensitivity; stability

\section{Introduction}

The main feature of individual SWNT sensors, besides their small size is that they operate at room temperature with higher sensitivity. SWNTs possess several properties that are very essential for gas sensors. They have all their atoms on the surface, endowing them with the highest specific surface area possible together with graphene. Therefore, all the carbon atoms in the nanotube can, in principle, interact with the analytic gas, while simultaneously supporting charge transport in the device. Thus, adsorbates and electrostatic charges and dipoles close to the nanotube can greatly impact charge transport. At the same time, the carbon nanotube lattice is held together by strong sp2 C-C bonds, which provide the necessary chemical stability to the carbon nanotube. An individual SWNTs sensor can be used to detect different types of molecules [1].

Detecting gas molecules is basic to environmental monitoring [2], control on chemical processing [3], space mission [4], agricultural and medical applications [1]. This type of device is very important because there are many gases which are harmful to organic life, such as humans and animals. One of the gases to be verified is nitrogen dioxide $\left(\mathrm{NO}_{2}\right)$. Even in small concentrations, it irritates the respiratory tract in large concentration causes pulmonary edema. $\mathrm{NO}_{2}$ create disturbance mainly in the airways and lungs, but also causes changes in blood composition, in particular, reduces the content of haemoglobin in blood. At low concentration of only $0.23 \mathrm{mg} / \mathrm{m}^{3}$, one feels the presence of this gas, but its adverse effects observed in healthy individuals at concentrations of $\mathrm{NO}_{2}$ in all $0.56 \mathrm{mg} / \mathrm{m}^{3}$, which is four times lower than the detection threshold. People with chronic lung diseases experience difficulty in breathing even at a concentration of $0.38 \mathrm{mg} / \mathrm{m}^{3}$. Among all harmful gasses, $\mathrm{NO}_{2}$ is a well-known toxic gas and air pollutant and monitoring its concentration is crucial for air quality monitoring. Prolonged exposure to low concentration of $\mathrm{NO}_{2}$ capable of causing several health hazards such as coronary artery disease as well as stroke [5-6]. The sensitivity of SWNTs towards $\mathrm{NO}_{2}$ at atmospheric temperature as reported [1] is particularly interesting. The sensing of $\mathrm{NO}_{2}$ is important to monitor environmental pollution resulting from combustion or automotive emission [7-8]. In recent times, the accidents in the oil, coal, gas industries has been increases, which claim the lives of hundreds of people. Every year many people lose their life due to hazardous gas leakage [1-4].

Many research groups have discussed sensing mechanism of $\mathrm{NO}_{2}$ based on CNT. In order to improves the sensing performance, and more challengingly, how to improve sensitivity of sensor for different gas species. One promising way is the functionalization [4, 9] of carbon nanotubes. Many characteristics of CNTs are superior to most other materials. Thus, for example, Young's modulus, which depends on the diameter and chirality of a CNT defect, can reach 1.8TPa, while when the conventional carbon fibres, it is comparable to 800GPa. The bulk compressibility of CNTs is quite high and amounts to 0.024GPa-1. If bent CNT also exhibit exceptional flexibility, their electrical conductivity depends on the magnetic field induction [10]. The magnetic properties of CNTs are remarkably different from the properties of diamond and graphite. The first measurements of the magnetic susceptibility showed that it greatly decreases with decreasing temperature of 300K. CNTs exhibit anisotropy magnetic property. With these properties, CNTs have broad application prospects, but their successful use is necessary to deal with some problems $[2,4,10]$. For example CNT through the possession of large surface energy, tend to form agglomerates, reaching up to tens or hundreds of micrometres. This leads to deterioration of the properties of CNTs in comparison with those that would be typical for homogeneous distribution. Solution to this problem can be achieved using various methods. CNT mechanical processing time must also be limited; since it increases the density of surface defects is increased [11]. Therefore, in addition to mechanical processing methods use the chemical treating CNTs to achieve more efficient dispersibility and impart additional properties. For example, using metal catalysts in the form of nanoparticles to decorate CNT, promotes the interaction with specific gas species. In this experiment CNTs have been functionalize by gold decoration. 
Existing gas sensors are based on metal oxide semiconductor. However they have a low sensitivity, high operating temperature and reaction time and substantial recovery. To ensure effective monitoring of air quality status it is necessary to improve the characteristics of gas sensors that can detect danger in advance. Development of $\mathrm{NO}_{2}$ sensors based on carbon nanotubes due to their unique properties will provide an opportunity to find a solution to these critical problems. To increase the sensitivity and selectivity to specific gas, as well as their reliability in various condition. The extraordinary property of SWNTs towards $\mathrm{NO}_{2}$ sensing attracts not only academicians but also industrials to make low power $\mathrm{NO}_{2}$ gas sensor. In present work, we are trying to solve above mentioned problems, for same, SWNTs grown sample is decorated with gold nanoparticles and also we have done detailed study on various effect of Au decoration on sensor characteristics.

\section{Experiment}

SWNTs used in this sensor have been grown by standard Chemical vapor Deposition (CVD) technique [10,13, 14]. CVD technique is one of the best technology for CNTs growth on silicon wafers.

We grow SWNTs on 5X5 mm chromium coated silicon wafer by standard CVD method [10, 13, 15]. Deposited SWNTs are decorated by gold. Gold is coated over sample by sputtering system. After that two electrodes are made by standard lithography technique as shown in Fig.1.

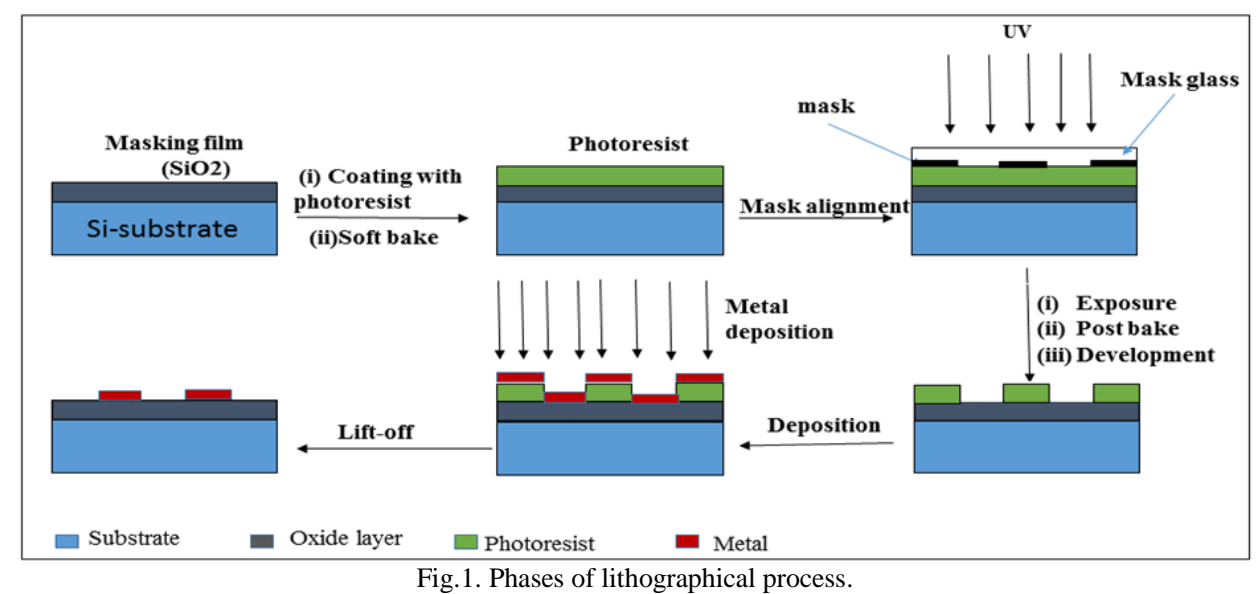

Formation of sensor electrode has the following successive processes: lithography, deposition and etching. Typical lithography process includes a set of operations that can be divided into three phases (Fig.1):

- $\quad$ Forming a continuous uniform layer of resist on the substrate surface;

- $\quad$ Once the surface has been coated with photoresist, the substrate is exposed to UV light;

- $\quad$ Once exposed, the substrate is immersed in a developer solution.

$\mathrm{NO}_{2}$ sensor research work carried out based on a CNT in a special chamber with one side connected to the gas distributor and on the other with the release into the environment. The gas supply comes from the two cylinders: the first bottle contain s only air, the second air cylinder $+\mathrm{NO}_{2}$ concentration of $100 \mathrm{ppm}$. The camera also has outputs for connection of an oscilloscope, multimeter that allows you to measure the change in resistance of the sensor in real time. Restoring the sensor is carried out by exposure to UV radiation. The flow of UV rays sent directly to the camera cell by limiting their distribution area. The calculation and measurement of the concentration of nitrogen dioxide $\left(\mathrm{NO}_{2}\right)$ to obtain the experimental data; regulation of the inlet gas concentration is done by standard mass flow controller and change in the resistance is measured by using multimeter/oscilloscope.

\section{Results and discussion}

Figure 2 shows the scanning electron microscopy (FESEM) image of pristine SWNTs grown over silicon substrate, in which we clearly observe a dense horizontal network of SWNTs over all substrate. The present SWNTs on substrate also verify by Raman spectroscopy (Fig.4). In Raman spectra, a sharp peak in the range of $200 \mathrm{~cm}-1$ to $300 \mathrm{~cm}-1$ is verifying the existence of SWNTs on silicon substrate. Figure 3 shows the FESEM image of Au decorated SWNTs surface, where we can see nonuniform particles of gold is distributed on every CNT. First we had done sensing experiment without UV supported recovery. And we found that the recovery time is more than 12 hours, which is impractical and does not meet all the tasks to be performed by the sensor. For the functional operation of the sensor it is necessary to its full recovery after each cycle of gas exposure. To expedite this process, we need to give the adsorbed gas molecules enough energy to break chemical bonds and their desorption from the surface of the CNTs. To achieve such an effect is possible by heating or exposing the sensor with UV exposure. Exposure to UV light is more advantageous way compared with heating, since, firstly, quantum energy UV radiation allows strong enough to destroy the chemical bonds, thereby accelerating the desorption process several times; Second, importantly, the use of UV lamps easier to operate [15-20]. After that we performed a series of experiment to monitor the response of the sensor with different concentrations of $\mathrm{NO}_{2}$, followed by reduction by means of UV radiation (see Fig.5 to Fig. 6). To see the various 
effect of gold decoration on sensing property firstly we perform the sensing experiment on pristine SWNTs with the concentration of $\mathrm{NO}_{2}$ is $40 \mathrm{ppm}$ level and we found initial resistance $\mathrm{Ri}=65.06 \mathrm{~K} \Omega$. After the start of gas supply to the resistance test chamber starts to decrease gradually. The response of the sensor is a $1 \sim 3$ seconds. After 5 minutes the gas flow was stopped, the camera only did the air flow and also produces ultraviolet light. Almost immediate increase in resistance was noted. Full recovery of the sensor to the initial position was 4 minutes 30 seconds. Now same experiment was repeated for gold decorated SWNTs sample with kept all sensing parameter same as before.

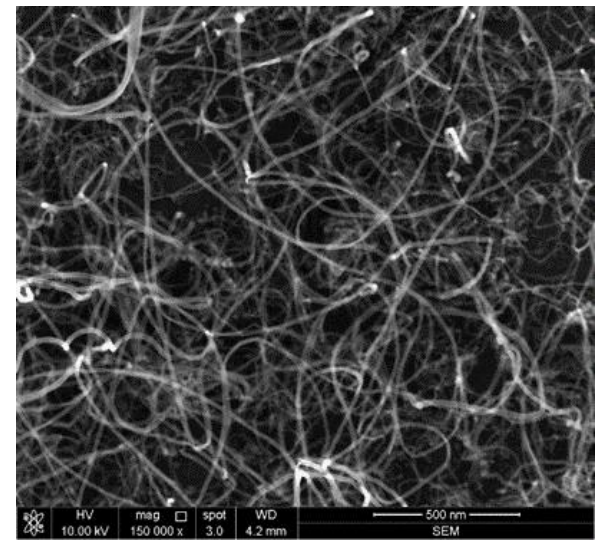

Fig. 2. FESEM image of pristine SWNTs.

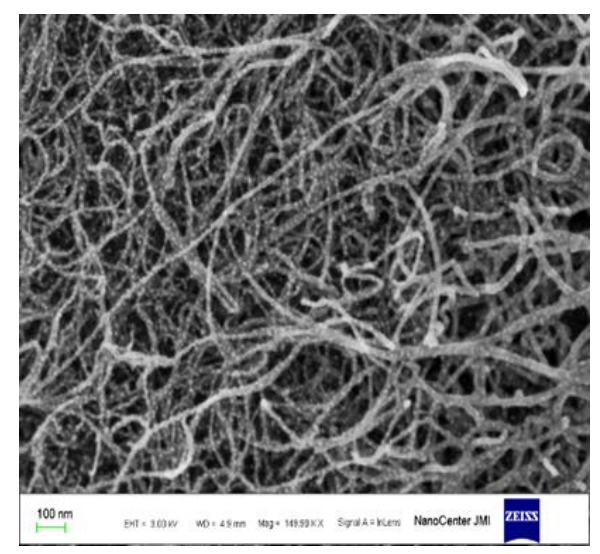

Fig. 3. FESEM image of gold decorated SWNTs.

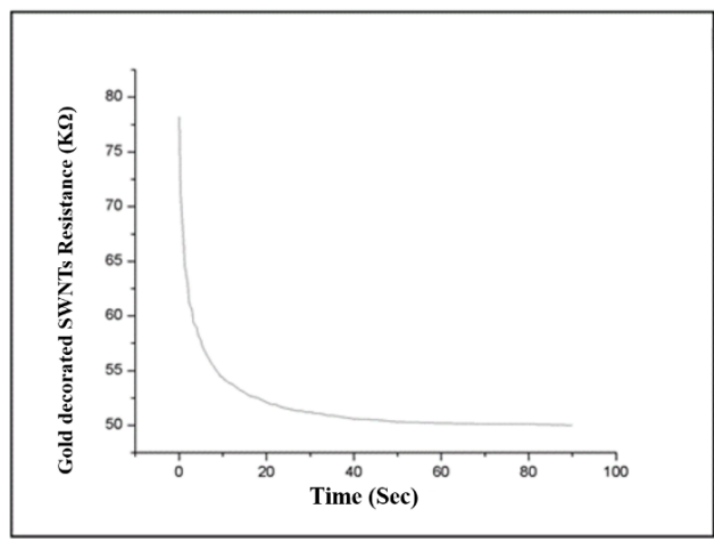

Fig. 4. Raman spectra of pristine SWNTs.

It is observed that initial resistance $\mathrm{Ri}=78.2 \mathrm{~K} \Omega$ sharply decreased to drag reduction occurred more rapidly than without gold sample. Comparison between $\mathrm{NO}_{2}$ gas sensor without gold coated and with gold coated has been shown in the Fig. 6 The sensitivity for each case can be calculated by formula:

$$
S=\frac{R_{0}-R_{N O_{2}}}{R_{0}} X 100 \%
$$

Where $-\mathrm{S}$ is the sensitivity of the sensor; $\mathrm{R}_{0}$ is the sensor resistance before you start working; $\mathrm{R}_{\mathrm{NO} 2}$ is the resistance of the sensor at the end of the experiment. And we found sensor sensitivity for pristine type sensor approximately 30percent and for gold decorated sensor around 38 percent approximately. The comparison between both type of sensor also shown in Fig. 6 and it is clearly observe from figure that gold decorated sensor have better sensitivity as compare to pristine SWNTs sensor. The possible reason for better sensitivity is that gold decorated CNTs have larger surface area as compare to pristine CNTs and hence the area for gas molecules interaction with sample is also larger. 


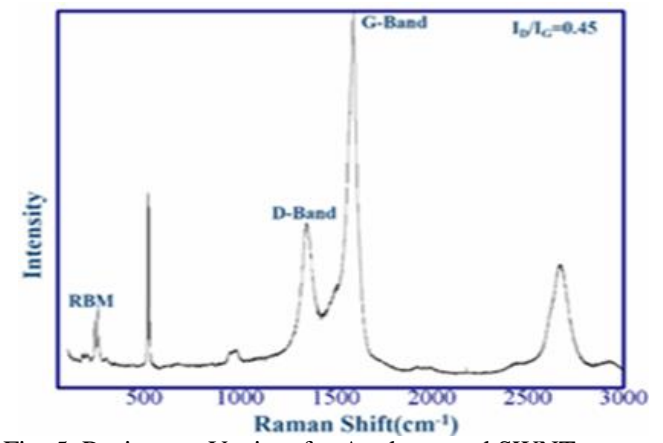

Fig. 5. Resistance Vs time for Au decorated SWNTs sensor.

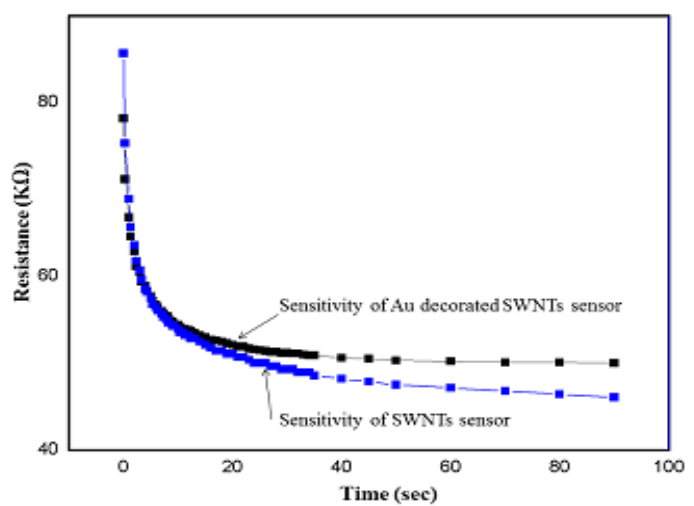

Fig. 6. Plot of the comparison between response of $\mathrm{SWNT} \mathrm{NO}_{2}$ sensor and Au-modified $\mathrm{SWNT} \mathrm{NO}_{2}$ sensor. Au-modified $\mathrm{SWNT} \mathrm{NO}_{2}$ sensor showed increase in sensitivity as compared with without gold coated $\mathrm{SWNT}_{\mathrm{NO}}$ sensor.

\section{Conclusion}

The conclusion of our work is that we successfully developed a good quality $\mathrm{NO}_{2}$ sensor. Based on the results of observations it can be argued that the sensor has an almost instantaneous reaction rate to the feed gas and the selected recovery technique using UV radiation has advantages over previous technologies by small time and ease of use. The change in sensitivity of SWNT sensor is induced by the coating of Au layer. The chemical pattern clearly demonstrates a significantly higher sensitivity of the Au-modified SWNT sensor compared with the un-functionalized SWNT sensor for $\mathrm{NO}_{2}$ gas.

\section{Reference}

[1] Kong J. Nanotube Molecular Wires as Chemical Sensors. Science 2000; 287(5453): 622-625.

[2] Zanolli Z, Leghrib R, Felten A, Pireaux J-J, Llobet E, Charlier J-C. Gas sensing with au-decorated carbon nanotubes. ACS Nano 2011; 5(6).

[3] Zeng Q, Luna J, Bayazitoglu Y, Wilson K, Imam MA, BarreraEV. Metal Coated Functionalized Single-Walled Carbon Nanotubes for Composites Application. Mater. Sci. Forum 2007; 561-565: 655-658.

[4] Sun Y-P, Fu K, Lin Y, Huang W. Functionalized Carbon Nanotubes: Properties and Applications. Acc. Chem. Res. 2002; $35(12)$ : $1096-1104$.

[5] Vallejos S, Grácia I, Chmela O, Figueras E, Hubálek J, Cané C. Chemoresistive micromachined gas sensors based on functionalized metal oxide nanowires: Performance and reliability. Sensors Actuators B Chem. 2016; 235: 525-534.

[6] Suehiro J, Zhou G, Hara M. Fabrication of a carbon nanotube-based gas sensor using dielectrophoresis and its application for ammonia detection by impedance spectroscopy. J. Phys. D. Appl. Phys. 2003; 36(21): L109- L114.

[7] Tran TH, Lee J-W, Lee K, Lee YD, Ju B-K. The gas sensing properties of single-walled carbon nanotubes deposited on an aminosilane monolayer. Sensors Actuators B Chem. 2008; 129(1): 67-71.

[8] Penza M, Rossi R, Alvisi M, Signore MA, Cassano G, Dimaio D, Pentassuglia R, Piscopiello E, Serra E, Falconieri M. Characterization of metal-modified and vertically-aligned carbon nanotube films for functionally enhanced gas sensor applications. Thin Solid Films 2009; 517(22).

[9] Derycke V, Auvray S, Borghetti J, Chung C-L, Lefèvre R, Lopez-Bezanilla A, Nguyen K, Robert G, Schmidt G, Anghel C, Chimot N, Lyonnais S, Streiff S, Campidelli S, Chenevier P, Filoramo A, Goffman MF, Goux-Capes L, Latil S, Blase X, Triozon F, Roche S, Bourgoin J-P. Carbon nanotube chemistry and assembly for electronic devices. Comptes Rendus Phys. 2009; 10(4): 330-347.

[10] Mishra P, Pavelyev VS, Patel R, Islam SS. Resistive sensing of gaseous nitrogen dioxide using a dispersion of single-walled carbon nanotubes in an ionic liquid. Mater. Res. Bull. 2016; 78: 53-57.

[11] Meng L, Fu C, Lu Q. Advanced technology for functionalization of carbon nanotubes. Prog. Nat. Sci. 2009; 19(7): 801-810.

[12]Lee K, Lee J-W, Dong K-Y, Ju B-K. Gas sensing properties of single-wall carbon nanotubes dispersed with dimethylformamide. Sensors Actuators B Chem. 2008; 135(1): 214-218.

[13] Tripathi N, Mishra P, Joshi B, Islam SS. Precise control over physical characteristics of Carbon Nanotubes by differential variation of Argon flow rate during Chemical Vapor Deposition processing: A systematic study on growth kinetics. Mater. Sci. Semicond. Process 2015; 35 : $207-215$.

[14] Tripathi N, Mishra P, Joshi B, Islam SS. Catalyst free, excellent quality and narrow diameter of CNT growth on Al2O3 by a thermal CVD technique. Phys. E Low-dimensional Syst. Nanostructures 2017; 62: 43-47.

[15]Penza M, Rossi R, Alvisi M, Cassano G, Serra E. Functional characterization of carbon nanotube networked films functionalized with tuned loading of Au nanoclusters for gas sensing applications. Sensors Actuators, B Chem. 2009; 140(1).

[16] Brahim S, Colbern S, Gump R, Grigorian L. Tailoring gas sensing properties of carbon nanotubes. J. Appl. Phys. 2008; 104(2): 24502.

[17] Mishra P, Harsh, Islam SS. Trace level ammonia sensing by SWCNTs (network/film) based resistive sensor using a simple approach in sensor development and design. Int. Nano Lett. 2013; 3(1): 46.

[18]Peng N, Zhang Q, Chow CL, Tan OK, Marzari N. Sensing mechanisms for carbon nanotube based NH3 gas detection.. Nano Lett. 2009 ; 9(4): 1626-1630.

[19]Huang XJ, Choi YK. Chemical sensors based on nanostructured materials. 2007; 122(2): 659-671.

[20] Van PTH, Thanh NH, Van Quang V, Van Duy N, Hoa ND, Van Hieu N. Scalable fabrication of high-performance NO2 gas sensors based on tungsten oxide nanowires by on-chip growth and RuO2-functionalization. ACS Appl. Mater. Interfaces 2014; 6(15): 12022-12030. 